\title{
Using Artificial Intelligence for COVID-19 Chest X-ray Diagnosis
}

\author{
Andrew A. Borkowski, MD; Narayan A. Viswanadhan, MD; L. Brannon Thomas, MD, PhD; Rodney D. Guzman; \\ Lauren A. Deland, RN; and Stephen M. Mastorides, MD
}

\begin{abstract}
Background: Coronavirus disease-19 (COVID-19), caused by a novel member of the coronavirus family, is a respiratory disease that rapidly reached pandemic proportions with high morbidity and mortality. In only a few months, it has had a dramatic impact on society and world economies. COVID-19 has presented numerous challenges to all aspects of health care, including reliable methods for diagnosis, treatment, and prevention. Initial efforts to contain the spread of the virus were hampered by the time required to develop reliable diagnostic methods. Artificial intelligence (Al) is a rapidly growing field of computer science with many applications for health care. Machine learning is a subset of $\mathrm{Al}$ that uses deep learning with neural network algorithms. It can recognize patterns and achieve complex computational tasks often far quicker and with increased precision than can humans.
\end{abstract}

Methods: In this article, we explore the potential for the simple and widely available chest X-ray (CXR) to be used with Al to diagnose COVID-19 reliably. Microsoft CustomVision is an automated image classification and object detection system that is a part of Microsoft Azure Cognitive Services. We utilized publicly available CXR images for patients with COVID-19 pneumonia, pneumonia from other etiologies, and normal CXRs as a dataset to train Microsoft CustomVision.

Results: Our trained model overall demonstrated $92.9 \%$ sensitivity (recall) and positive predictive value (precision), with results for each label showing sensitivity and positive predictive value at $94.8 \%$ and $98.9 \%$ for COVID-19 pneumonia, $89 \%$ and $91.8 \%$ for non-COVID-19 pneumonia, 95\% and $88.8 \%$ for normal lung. We then validated the program using CXRs of patients from our institution with confirmed COVID-19 diagnoses along with non-COVID-19 pneumonia and normal CXRs. Our model performed with 100\% sensitivity, 95\% specificity, $97 \%$ accuracy, $91 \%$ positive predictive value, and $100 \%$ negative predictive value.

Conclusions: We have used a readily available, commercial platform to demonstrate the potential of Al to assist in the successful diagnosis of COVID-19 pneumonia on CXR images. The findings have implications for screening and triage, initial diagnosis, monitoring disease progression, and identifying patients at increased risk of morbidity and mortality. Based on the data, a website was created to demonstrate how such technologies could be shared and distributed to others to combat entities such as COVID-19 moving forward.
Author affiliations can be found at the end of the article.

Correspondence:

Andrew Borkowski

(andrew.borkowski@va.gov)

Fed Pract. 2020;37(9):398-404. doi: $10.12788 /$ fp. 0045
$\mathrm{T}$ he novel coronavirus severe acute respiratory syndrome coronavirus 2 (SARSCoV-2), which causes the respiratory disease coronavirus disease-19 (COVID19), was first identified as a cluster of cases of pneumonia in Wuhan, Hubei Province of China on December 31, 2019. ${ }^{1}$ Within a month, the disease had spread significantly, leading the World Health Organization (WHO) to designate COVID-19 a public health emergency of international concern. On March 11, 2020, the WHO declared COVID-19 a global pandemic. ${ }^{2}$ As of August 18,2020 , the virus has infected $>21$ million people, with $>750,000$ deaths worldwide. ${ }^{3}$ The spread of COVID-19 has had a dramatic impact on social, economic, and health care issues throughout the world, which has been discussed elsewhere. ${ }^{4}$

Prior to the this century, members of the coronavirus family had minimal impact on human health. ${ }^{5}$ However, in the past 20 years, outbreaks have highlighted an emerging importance of coronaviruses in morbidity and mortality on a global scale.
Although less prevalent than COVID-19, severe acute respiratory syndrome (SARS) in 2002 to 2003 and Middle East respiratory syndrome (MERS) in 2012 likely had higher mortality rates than the current pandemic. ${ }^{5}$ Based on this recent history, it is reasonable to assume that we will continue to see novel diseases with similar significant health and societal implications. The challenges presented to health care providers (HCPs) by such novel viral pathogens are numerous, including methods for rapid diagnosis, prevention, and treatment. In the current study, we focus on diagnosis issues, which were evident with COVID-19 with the time required to develop rapid and effective diagnostic modalities.

We have previously reported the utility of using artificial intelligence (AI) in the histopathologic diagnosis of cancer. ${ }^{6-8}$ AI was first described in 1956 and involves the field of computer science in which machines are trained to learn from experience. ${ }^{9}$ Machine learning (ML) is a subset of AI and is achieved by using mathematic 
models to compute sample datasets. ${ }^{10}$ Current ML employs deep learning with neural network algorithms, which can recognize patterns and achieve complex computational tasks often far quicker and with increased precision than can humans. ${ }^{11-13}$ In addition to applications in pathology, ML algorithms have both prognostic and diagnostic applications in multiple medical specialties, such as radiology, dermatology, ophthalmology, and cardiology. ${ }^{6}$ It is predicted that AI will impact almost every aspect of health care in the future. ${ }^{14}$

In this article, we examine the potential for AI to diagnose patients with COVID-19 pneumonia using chest radiographs (CXR) alone. This is done using Microsoft CustomVision (www.customvision.ai), a readily available, automated ML platform. Employing $\mathrm{AI}$ to both screen and diagnose emerging health emergencies such as COVID-19 has the potential to dramatically change how we approach medical care in the future. In addition, we describe the creation of a publicly available website (interknowlogy-covid-19 .azurewebsites.net) that could augment COVID-19 pneumonia CXR diagnosis.

\section{METHODS}

For the training dataset, 103 CXR images of COVID-19 were downloaded from GitHub covid-chest-xray dataset. ${ }^{15}$ Five hundred images of non-COVID-19 pneumonia and 500 images of the normal lung were downloaded from the Kaggle RSNA Pneumonia Detection Challenge dataset. ${ }^{16}$ To balance the dataset, we expanded the COVID-19 dataset to 500 images by slight rotation (probability $=1$, max rotation $=5$ ) and zooming (probability $=0.5$, percentage area $=0.9$ ) of the original images using the Augmentor Python package. ${ }^{17}$

\section{Validation Dataset}

For the validation dataset 30 random CXR images were obtained from the US Department of Veterans Affairs (VA) PACS (picture archiving and communication system). This dataset included 10 CXR images from hospitalized patients with COVID-19, 10 CXR pneumonia images from patients without COVID-19, and 10 normal CXRs. COVID-19 diagnoses were confirmed with a positive test result from the Xpert Xpress
TABLE Artificial Intelligence Algorithm Determinations ${ }^{a}$

\begin{tabular}{|c|c|c|c|c|}
\hline X-ray Types & COVID-19 & Non-COVID-19 & Normal & Determination \\
\hline \multicolumn{5}{|l|}{ Normal } \\
\hline 1 & 0 & 0 & 1 & True negative \\
\hline 2 & 0 & 0 & 1 & True negative \\
\hline 3 & 0 & 0 & 1 & True negative \\
\hline 4 & 0 & 0 & 1 & True negative \\
\hline 5 & 0 & 0 & 1 & True negative \\
\hline 6 & 0 & 0 & 1 & True negative \\
\hline 7 & 0 & 0 & 1 & True negative \\
\hline 8 & 0 & 0 & 1 & True negative \\
\hline 9 & 0 & 0 & 1 & True negative \\
\hline 10 & 0 & 0 & 1 & True negative \\
\hline \multicolumn{5}{|l|}{ COVID-19 } \\
\hline 1 & 1 & 0 & 0 & True positive \\
\hline 2 & 1 & 0 & 0 & True positive \\
\hline 3 & 1 & 0 & 0 & True positive \\
\hline 4 & 1 & 0 & 0 & True positive \\
\hline 5 & 1 & 0 & 0 & True positive \\
\hline 6 & 1 & 0 & 0 & True positive \\
\hline 7 & 1 & 0 & 0 & True positive \\
\hline 8 & 1 & 0 & 0 & True positive \\
\hline 9 & 1 & 0 & 0 & True positive \\
\hline 10 & 1 & 0 & 0 & True positive \\
\hline \multicolumn{5}{|c|}{ Non-COVID-19 } \\
\hline 1 & 0 & 1 & 0 & True negative \\
\hline 2 & 0 & 1 & 0 & True negative \\
\hline 3 & 0 & 1 & 0 & True negative \\
\hline 4 & 0 & 1 & 0 & False positive \\
\hline 5 & 1 & 0 & 0 & True negative \\
\hline 6 & 0 & 1 & 0 & True negative \\
\hline 7 & 0 & 1 & 0 & True negative \\
\hline 8 & 0 & 1 & 0 & True negative \\
\hline 9 & 0 & 0 & 1 & True negative \\
\hline 10 & 0 & 1 & 0 & True negative \\
\hline
\end{tabular}

\begin{tabular}{lclc} 
Sensitivity (recall) & $100 \%$ & True positive & 10 \\
\hline Specificity & $95 \%$ & True negative & 19 \\
\hline Accuracy & $97 \%$ & False positive & 1 \\
\hline Positive predictive value (precision) & $91 \%$ & False negative & 0 \\
\hline $\begin{array}{l}\text { Negative predictive value } \\
\text { a1 }=\text { positive; } 0=\text { negative }\end{array}$ & $100 \%$ & & \\
\hline
\end{tabular}

SARS-CoV-2 polymerase chain reaction (PCR) platform. ${ }^{18}$

\section{Microsoft CustomVision}

Microsoft CustomVision is an automated image classification and object detection system that is a part of Microsoft Azure Cognitive Services (azure.microsoft.com). It has a pay-as-you-go model with fees depending on the computing needs and usage. It offers a free trial to users for 2 initial projects. The service is online with an 
FIGURE 1 Image Dataset Used to Train Artificial Intelligence

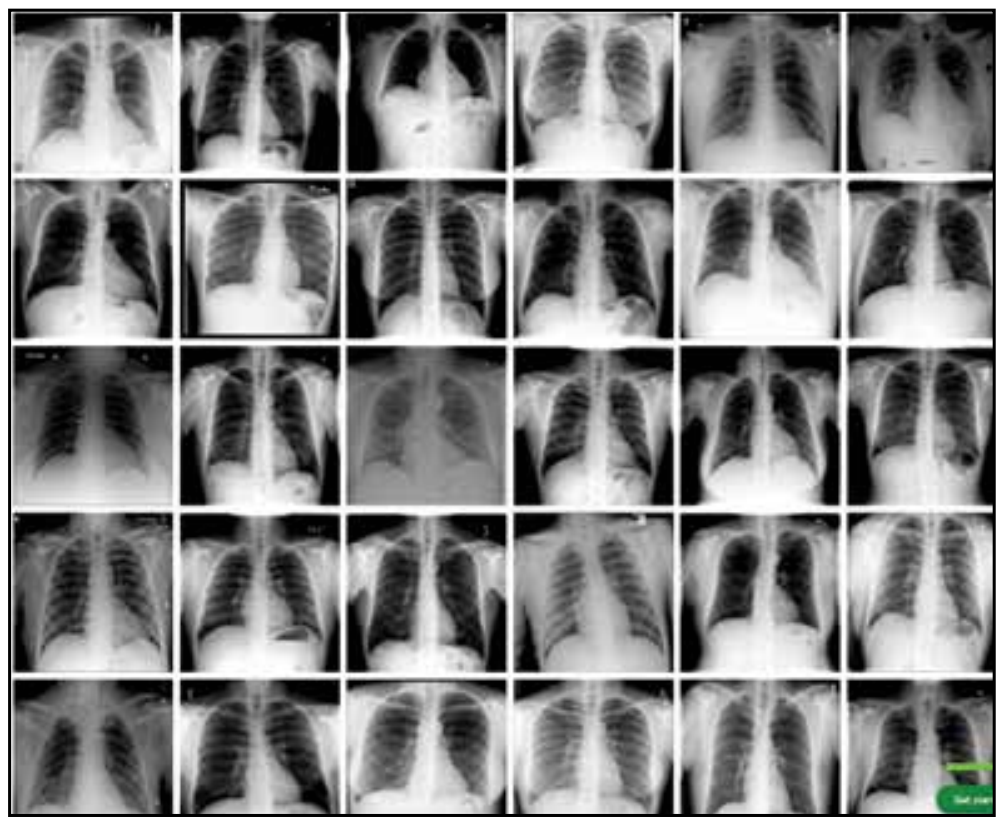

easy-to-follow graphical user interface. No coding skills are necessary.

We created a new classification project in CustomVision and chose a compact general domain for small size and easy export to TensorFlow.js model format. TensorFlow.js is a JavaScript library that enables dynamic download and execution of ML models. After the project was created, we proceeded to upload our image dataset. Each class was uploaded separately and tagged with the appropriate label (covid pneumonia, non-covid pneumonia, or normal lung). The system rejected 16 COVID-19 images as duplicates. The final CustomVision training dataset consisted of 484 images of COVID-19 pneumonia, 500 images of non-COVID-19 pneumonia, and 500 images of normal lungs. Once uploaded, CustomVision self-trains using the dataset upon initiating the program (Figure 1).

\section{Website Creation}

CustomVision was used to train the model. It can be used to execute the model continuously, or the model can be compacted and decoupled from CustomVision. In this case, the model was compacted and decoupled for use in an online application. An Angular online application was created with TensorFlow.js. Within a user's web browser, the model is executed when an image of a
CXR is submitted. Confidence values for each classification are returned. In this design, after the initial webpage and model is downloaded, the webpage no longer needs to access any server components and performs all operations in the browser. Although the solution works well on mobile phone browsers and in low bandwidth situations, the quality of predictions may depend on the browser and device used. At no time does an image get submitted to the cloud.

\section{RESULTS}

Overall, our trained model showed 92.9\% precision and recall. Precision and recall results for each label were $98.9 \%$ and $94.8 \%$, respectively for COVID-19 pneumonia; $91.8 \%$ and $89 \%$, respectively, for nonCOVID-19 pneumonia; and $88.8 \%$ and $95 \%$, respectively, for normal lung (Figure 2). Next, we proceeded to validate the training model on the VA data by making individual predictions on 30 images from the VA dataset. Our model performed well with $100 \%$ sensitivity (recall), 95\% specificity, 97\% accuracy, 91\% positive predictive value (precision), and $100 \%$ negative predictive value (Table).

\section{DISCUSSION}

We successfully demonstrated the potential of using AI algorithms in assessing CXRs for COVID-19. We first trained the CustomVision automated image classification and object detection system to differentiate cases of COVID-19 from pneumonia from other etiologies as well as normal lung CXRs. We then tested our model against known patients from the James A. Haley Veterans' Hospital in Tampa, Florida. The program achieved 100\% sensitivity (recall), 95\% specificity, $97 \%$ accuracy, $91 \%$ positive predictive value (precision), and $100 \%$ negative predictive value in differentiating the 3 scenarios. Using the trained ML model, we proceeded to create a website that could augment COVID-19 CXR diagnosis. ${ }^{19}$ The website works on mobile as well as desktop platforms. A health care provider can take a CXR photo with a mobile phone or upload the image file. The ML algorithm would provide the probability of COVID-19 pneumonia, non-COVID-19 pneumonia, or normal lung diagnosis (Figure 3). 
Emerging diseases such as COVID-19 present numerous challenges to HCPs, governments, and businesses, as well as to individual members of society. As evidenced with COVID-19, the time from first recognition of an emerging pathogen to the development of methods for reliable diagnosis and treatment can be months, even with a concerted international effort. The gold standard for diagnosis of COVID-19 is by reverse transcriptase PCR (RT-PCR) technologies; however, early RT-PCR testing produced less than optimal results. ${ }^{20-22}$ Even after the development of reliable tests for detection, making test kits readily available to health care providers on an adequate scale presents an additional challenge as evident with COVID-19.

\section{Use of X-ray vs Computed Tomography}

The lack of availability of diagnostic RTPCR with COVID-19 initially placed increased reliability on presumptive diagnoses via imaging in some situations. ${ }^{23}$ Most of the literature evaluating radiographs of patients with COVID-19 focuses on chest computed tomography (CT) findings, with initial results suggesting CT was more accurate than early RT-PCR methodologies..$^{21,22,24}$ The Radiological Society of North America Expert consensus statement on chest CT for COVID-19 states that CT findings can even precede positivity on RT-PCR in some cases. ${ }^{22}$ However, currently it does not recommend the use of CT scanning as a screening tool. Furthermore, the actual sensitivity and specificity of CT interpretation by radiologists for COVID-19 are unknown. ${ }^{22}$

Characteristic CT findings include ground-glass opacities (GGOs) and consolidation most commonly in the lung periphery, though a diffuse distribution was found in a minority of patients. ${ }^{21,23,25-27}$ Lomoro and colleagues recently summarized the CT findings from several reports that described abnormalities as most often bilateral and peripheral, subpleural, and affecting the lower lobes. ${ }^{26}$ Not surprisingly, CT appears more sensitive at detecting changes with COVID-19 than does CXR, with reports that a minority of patients exhibited CT changes before changes were visible on CXR. ${ }^{23,26}$

We focused our study on the potential of AI in the examination of CXRs in patients with COVID-19, as there are several limi-

\section{FIGURE 2 Trained Machine Learning Model Performance Example}

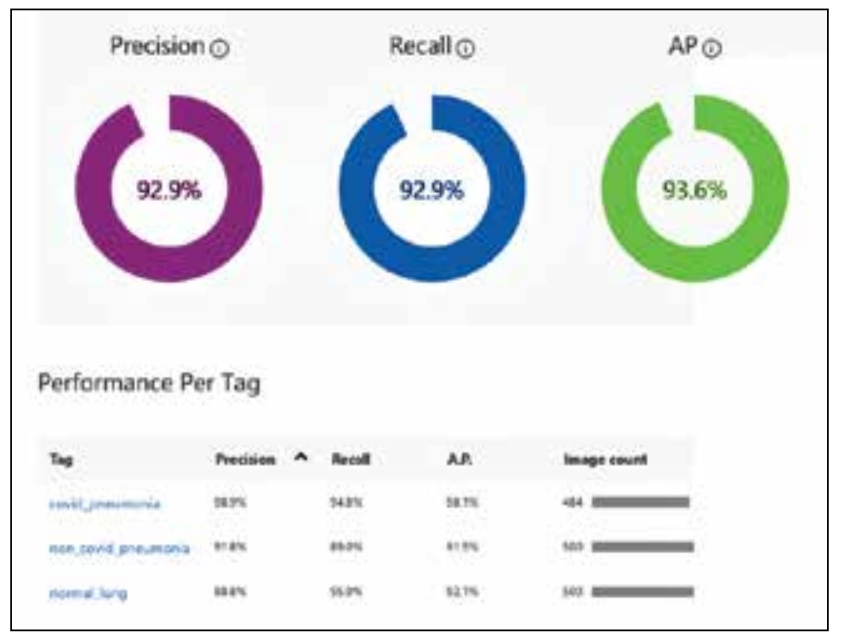

tations to the routine use of CT scans with conditions such as COVID-19. Aside from the more considerable time required to obtain CTs, there are issues with contamination of CT suites, sometimes requiring a dedicated COVID-19 CT scanner. ${ }^{23,28}$ The time constraints of decontamination or limited utilization of CT suites can delay or disrupt services for patients with and without COVID-19. Because of these factors, CXR may be a better resource to minimize the risk of infection to other patients. Also, accurate assessment of abnormalities on CXR for COVID-19 may identify patients in whom the CXR was performed for other purposes. ${ }^{23} \mathrm{CXR}$ is more readily available than $\mathrm{CT}$, especially in more remote or underdeveloped areas. ${ }^{28}$ Finally, as with CT, CXR abnormalities are reported to have appeared before RT-PCR tests became positive for a minority of patients. ${ }^{23}$

CXR findings described in patients with COVID-19 are similar to those of CT and include GGOs, consolidation, and hazy increased opacities. ${ }^{23,25,26,28,29}$ Like CT, the majority of patients who received CXR demonstrated greater involvement in the lower zones and peripherally. ${ }^{23,25,26,28,29}$ Most patients showed bilateral involvement. However, while these findings are common in patients with COVID-19, they are not specific and can be seen in other conditions, such as other viral pneumonia, bacterial pneumonia, injury from drug toxicity, inhalation injury, connective tissue disease, and idiopathic conditions. 
FIGURE 3 Artificial Intelligence Research Website

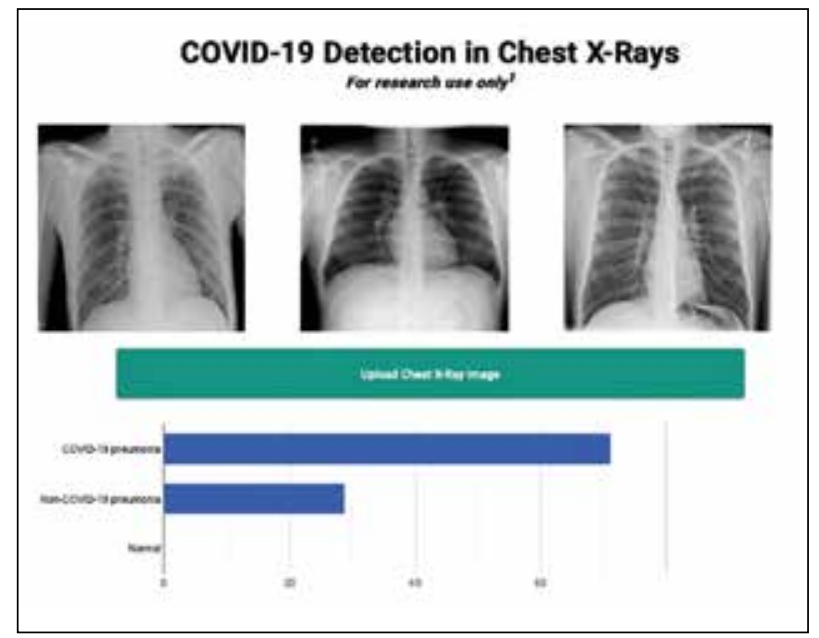

Application of Al for COVID-19

Applications of AI in interpreting radiographs of various types are numerous, and extensive literature has been written on the topic. ${ }^{30}$ Using deep learning algorithms, AI has multiple possible roles to augment traditional radiograph interpretation. These include the potential for screening, triaging, and increasing the speed to render diagnoses. It also can provide a rapid "second opinion" to the radiologist to support the final interpretation. In areas with critical shortages of radiologists, AI potentially can be used to render the definitive diagnosis. In COVID19, imaging studies have been shown to correlate with disease severity and mortality, and AI could assist in monitoring the course of the disease as it progresses and potentially identify patients at greatest risk. ${ }^{27}$ Furthermore, early results from PCR have been considered suboptimal, and it is known that patients with COVID-19 can test negative initially even by reliable testing methodologies. As AI technology progresses, interpretation can detect and guide triage and treatment of patients with high suspicions of COVID-19 but negative initial PCR results, or in situations where test availability is limited or results are delayed. There are numerous potential benefits should a rapid diagnostic test as simple as a CXR be able to reliably impact containment and prevention of the spread of contagions such as COVID19 early in its course.

Few studies have assessed using AI in the radiologic diagnosis of COVID-19, most of which use CT scanning. Bai and colleagues demonstrated increased accuracy, sensitivity, and specificity in distinguishing chest CTs of COVID-19 patients from other types of pneumonia. ${ }^{21,31}$ A separate study demonstrated the utility of using AI to differentiate COVID-19 from community-acquired pneumonia with $\mathrm{CT}^{32}$ However, the effective utility of AI for CXR interpretation also has been demonstrated. ${ }^{14,33}$ Implementation of convolutional neural network layers has allowed for reliable differentiation of viral and bacterial pneumonia with CXR imaging. ${ }^{34}$ Evidence suggests that there is great potential in the application of $\mathrm{AI}$ in the interpretation of radiographs of all types.

Finally, we have developed a publicly available website based on our studies. ${ }^{18}$ This website is for research use only as it is based on data from our preliminary investigation. To appear within the website, images must have protected health information removed before uploading. The information on the website, including text, graphics, images, or other material, is for research and may not be appropriate for all circumstances. The website does not provide medical, professional, or licensed advice and is not a substitute for consultation with a HCP. Medical advice should be sought from a qualified HCP for any questions, and the website should not be used for medical diagnosis or treatment.

\section{Limitations}

In our preliminary study, we have demonstrated the potential impact AI can have in multiple aspects of patient care for emerging pathogens such as COVID-19 using a test as readily available as a CXR. However, several limitations to this investigation should be mentioned. The study is retrospective in nature with limited sample size and with X-rays from patients with various stages of COVID-19 pneumonia. Also, cases of non-COVID-19 pneumonia are not stratified into different types or etiologies. We intend to demonstrate the potential of AI in differentiating COVID-19 pneumonia from non-COVID-19 pneumonia of any etiology, though future studies should address comparison of COVID-19 cases to more specific types of pneumonias, such as of bacterial or 
viral origin. Furthermore, the present study does not address any potential effects of additional radiographic findings from coexistent conditions, such as pulmonary edema as seen in congestive heart failure, pleural effusions (which can be seen with COVID19 pneumonia, though rarely), interstitial lung disease, etc. Future studies are required to address these issues. Ultimately, prospective studies to assess AI-assisted radiographic interpretation in conditions such as COVID-19 are required to demonstrate the impact on diagnosis, treatment, outcome, and patient safety as these technologies are implemented.

\section{CONCLUSIONS}

We have used a readily available, commercial platform to demonstrate the potential of AI to assist in the successful diagnosis of COVID-19 pneumonia on CXR images. While this technology has numerous applications in radiology, we have focused on the potential impact on future world health crises such as COVID-19. The findings have implications for screening and triage, initial diagnosis, monitoring disease progression, and identifying patients at increased risk of morbidity and mortality. Based on the data, a website was created to demonstrate how such technologies could be shared and distributed to others to combat entities such as COVID-19 moving forward. Our study offers a small window into the potential for how AI will likely dramatically change the practice of medicine in the future.

\section{Acknowledgments}

This material is the result of work supported with resources and the use of facilities at the James A. Haley Veterans' Hospital and computational resources of the InterKnowlogy, LLC. This activity has been approved by the James A. Haley Veterans' Hospital Office of Research and Development.

\section{Author affiliations}

Andrew Borkowski is Chief of the Molecular Diagnostics Laboratory, L. Brannon Thomas is Chief of the Microbiology Laboratory, Lauren Deland is a Research Coordinator, and Stephen Mastorides is Chief of Pathology; Narayan Viswanadhan is Assistant Chief of Radiology; all at the James A. Haley Veterans' Hospital in Tampa, Florida. Rodney Guzman is a Cofounder of InterKnowlogy, LLC in Carlsbad, California. Andrew Borkowski and Stephen Mastorides are Professors and L. Brannon Thomas is an Assistant Professor, all in the Department of Pathology and Cell Biology, University of South Florida, Morsani College of Medicine in Tampa, Florida

\section{Author disclosures}

The authors report no actual or potential conflicts of interest with regard to this article.

\section{Disclaimer}

The opinions expressed herein are those of the authors and do not necessarily reflect those of Federal Practitioner, Frontline Medical Communications Inc., the US Government, or any of its agencies.

\section{References}

1. World Health Organization. Coronavirus disease (COVID19) pandemic. https://www.who.int/emergencies/diseases /novel-coronavirus2019. Updated August 23, 2020. Accessed August 24, 2020.

2. World Health Organization. WHO Director-General's opening remarks at the media briefing on COVID-19 - 11 March 2020. https://www.who.int/dg/speeches/detail/who -director-general-sopening-remarks-at-the-media-briefing -on-covid-19---11-march2020. Published March 11, 2020. Accessed August 24, 2020.

3. World Health Organization. Coronavirus disease (COVID19): situation report--209. https://www.who.int/docs /default-source/coronaviruse/situation-reports/20200816 -covid-19-sitrep-209.pdf. Updated August 16, 2020. Accessed August 24, 2020.

4. Nicola M, Alsafi Z, Sohrabi C, et al. The socio-economic implications of the coronavirus pandemic (COVID-19): a review. Int J Surg. 2020;78:185-193. doi:10.1016/j.jisu.2020.04.018

5. da Costa VG, Moreli ML, Saivish MV. The emergence of SARS, MERS and novel SARS-2 coronaviruses in the 21st century. Arch Virol. 2020;165(7):1517-1526. doi:10.1007/s00705-020-04628-0

6. Borkowski AA, Wilson CP, Borkowski SA, et al. Comparing artificial intelligence platforms for histopathologic cancer diagnosis. Fed Pract. 2019;36(10):456-463.

7. Borkowski AA, Wilson CP, Borkowski SA, Thomas LB, Deland LA, Mastorides SM. Apple machine learning algorithms successfully detect colon cancer but fail to predict KRAS mutation status. http://arxiv.org/abs/1812.04660. Updated January 15, 2019. Accessed August 24, 2020.

8. Borkowski AA, Wilson CP, Borkowski SA, Deland LA, Mastorides SM. Using Apple machine learning algorithms to detect and subclassify non-small cell lung cancer. http:// arxiv.org/abs/1808.08230. Updated January 15, 2019. Accessed August 24, 2020.

9. Moor J. The Dartmouth College artificial intelligence conference: the next fifty years. Al Mag. 2006;27(4):87. doi:10.1609/AIMAG.V2714.1911

10. Samuel AL. Some studies in machine learning using the game of checkers. IBM J Res Dev. 1959;3(3):210-229. doi:10.1147/rd.33.0210

11. Sarle WS. Neural networks and statistical models https:// people.orie.cornell.edu/davidr/or474/nn_sas.pdf. Published April 1994. Accessed August 24, 2020.

12. Schmidhuber J. Deep learning in neural networks: an overview. Neural Netw. 2015;61:85-117. doi:10.1016/j.neunet.2014.09.003

13. LeCun $Y$, Bengio $Y$, Hinton $G$. Deep learning. Nature 2015;521(7553):436-444. doi:10.1038/nature14539

14. Topol EJ. High-performance medicine: the convergence of human and artificial intelligence. Nat Med. 2019;25(1):4456. doi:10.1038/s41591-018-0300-7

15. Cohen JP, Morrison P, Dao L. COVID-19 Image Data Collection. Published online March 25, 2020. Accessed May 13, 2020. http://arxiv.org/abs/2003.11597

16. Radiological Society of America. RSNA pneumonia detection challenge. https://www.kaggle.com/c/rsnapneumonia-detectionchallenge. Accessed August 24, 2020.

17. Bloice MD, Roth PM, Holzinger A. Biomedical image augmentation using Augmentor. Bioinformatics. 2019;35(21):4522-4524. doi:10.1093/bioinformatics/btz259

18. Cepheid. Xpert Xpress SARS-CoV-2. https://www.cepheid .com/coronavirus. Accessed August 24, 2020.

19. Interknowlogy. COVID-19 detection in chest X-rays. https://interknowlogy-covid-19.azurewebsites.net. Ac- 
cessed August 27, 2020.

20. Bernheim A, Mei X, Huang M, et al. Chest CT Findings in Coronavirus Disease-19 (COVID-19): Relationship to Duration of Infection. Radiology. 2020;295(3):200463. doi:10.1148/radiol.2020200463

21. Ai T, Yang Z, Hou H, et al. Correlation of Chest CT and RTPCR Testing for Coronavirus Disease 2019 (COVID-19) in China: a report of 1014 cases. Radiology. 2020;296(2):E32E40. doi:10.1148/radiol.2020200642

22. Simpson S, Kay FU, Abbara S, et al. Radiological Society of North America Expert Consensus Statement on Reporting Chest CT Findings Related to COVID-19. Endorsed by the Society of Thoracic Radiology, the American College of Radiology, and RSNA - Secondary Publication. J Thorac Imaging. 2020;35(4):219-227. doi:10.1097/RTI.0000000000000524

23. Wong HYF, Lam HYS, Fong AH, et al. Frequency and distribution of chest radiographic findings in patients positive for COVID-19. Radiology. 2020;296(2):E72-E78. doi:10.1148/radiol.2020201160

24. Fang $\mathrm{Y}$, Zhang $\mathrm{H}, \mathrm{Xie} \mathrm{J}$, et al. Sensitivity of chest $\mathrm{CT}$ for COVID-19: comparison to RT-PCR. Radiology. 2020;296(2):E115-E117. doi:10.1148/radiol.2020200432

25. Chen N, Zhou M, Dong X, et al. Epidemiological and clinical characteristics of 99 cases of 2019 novel coronavirus pneumonia in Wuhan, China: a descriptive study. Lancet. 2020;395(10223):507-513. doi:10.1016/S0140-6736(20)30211-7

26. Lomoro P, Verde F, Zerboni F, et al. COVID-19 pneumonia manifestations at the admission on chest ultrasound, radiographs, and $\mathrm{CT}$ : single-center study and comprehensive radiologic literature review. Eur J Radiol Open. 2020;7:100231. doi:10.1016/j.ejro.2020.100231

27. Salehi S, Abedi A, Balakrishnan S, Gholamrezanezhad A. Coronavirus disease 2019 (COVID-19) imaging reporting and data system (COVID-RADS) and common lexicon: a proposal based on the imaging data of 37 studies. Eur Radiol. 2020;30(9):4930-4942. doi:10.1007/s00330-020-06863-0

28. Jacobi A, Chung M, Bernheim A, Eber C. Portable chest X-ray in coronavirus disease-19 (COVID19): a pictorial review. Clin Imaging. 2020;64:35-42. doi:10.1016/j.clinimag.2020.04.001

29. Bhat R, Hamid A, Kunin JR, et al. Chest imaging in patients hospitalized With COVID-19 infection - a case series. Curr Probl Diagn Radiol. 2020;49(4):294-301. doi:10.1067/j.cpradiol.2020.04.001

30. Liu X, Faes L, Kale AU, et al. A comparison of deep learning performance against health-care professionals in detecting diseases from medical imaging: a systematic review and meta-analysis. Lancet Digit Heal. 2019;1(6):E271E297. doi:10.1016/S2589-7500(19)30123-2

31. Bai $H X$, Wang $R$, Xiong $Z$, et al. Artificial intelligence augmentation of radiologist performance in distinguishing COVID-19 from pneumonia of other origin at chest CT. Radiology. 2020;296(3):E156-E165. doi:10.1148/radiol.2020201491

32. Li L, Qin L, Xu Z, et al. Using artificial intelligence to detect COVID-19 and community-acquired pneumonia based on pulmonary CT: evaluation of the diagnostic accuracy. Radiology. 2020;296(2):E65-E71. doi:10.1148/radiol.2020200905

33. Rajpurkar P, Joshi A, Pareek A, et al. CheXpedition: investigating generalization challenges for translation of chest x-ray algorithms to the clinical setting. http://arxiv.org /abs/2002.11379. Updated March 11, 2020. Accessed August 24, 2020.

34. Kermany DS, Goldbaum M, Cai W, et al. Identifying medical diagnoses and treatable diseases by imagebased deep learning. Cell. 2018;172(5):1122-1131.e9. doi:10.1016/j.cell.2018.02.010 